San Jose State University

From the SelectedWorks of Brian Belet

2008

\title{
Theoretical and Formal Continuity in James Tenney's Music
}

Brian Belet, San Jose State University 


\title{
Theoretical and Formal Continuity in James Tenney's Music
}

\author{
Dr. Brian Belet \\ School of Music and Dance \\ San Jose State University, San Jose, California, 95192-0095, USA
}

\begin{abstract}
James Tenney created much of his music, and his theoretical writings, as an objective experimenter, observer, and codifier. This paper will examine Tenney's traits of curiosity, experimentation, and honest self-evaluation through a subset of his compositions primarily from the 1960s, 1970s, and 1980s. Although quite diverse in many ways these compositions retain his mark of intense individuality, integrity, and compositional rigor, which creates a macro unity and formal continuity. Perhaps this is his ultimate super "clang" and conceptual "temporal gestalt unit." Each composition grows out of the need to address one or more specific formal questions: each work is indeed an experiment designed to explore the inherent ramifications of a theory, and subsequent compositions addressed questions generated by earlier works. Reclaiming the term "theorist" in the proper sense within music, James Tenney designed and refined theories that required his compositions to prove or disprove them. In this way each composition is linked to the next, and this continuous exploration established his large-scale formal and philosophical continuity of his creative life.
\end{abstract}

\subsection{Formal Continuity: Aesthetics, Theory, and Practice}

Twenty years ago Budd (1987) declared that James Tenney "is that most dangerous man imaginable in art. He knows he's right, and then shows you why. That, incidentally, is my definition of a theorist." At about the same time, when asked by this author "Why do you compose?" (Belet, 1987, p. 459) Tenney replied without hesitation: "Curiosity, I think, is the strongest motivating force of almost everything I do."

The clarity and immediacy of his response demonstrated that he had asked himself this very question, not just once and then shelved, but repeatedly and in depth. His answer was a personal statement of compositional purpose and balance, a guiding aesthetic to inform and guide all of his composing and theoretical writing. Tenney's motivating curiosity linked his life-long interest in science to his art. While science is primarily concerned with understanding reality using thought and intellectual ordering, art is more concerned with using perception to explore and understand our reality. The need to understand creates the common ground between the two pursuits. Tenney described his personal view of this connection (Belet, 1987, p. 459): “They are

Contemporary Music Review, Vol. 27, No. 1, Routledge (London), February 2008, pp. 23-45.

(C) 2007 Brian Belet - All Rights Reserved 
two manifestations of a curiosity. I often think about the pieces that I compose: I write them because I want to know what they are going to sound like.” Earlier, in a conversation with Kasemets, Pearson, and Monahan (1984, p. 3), Tenney elaborated:

I think we're all phenomenologists. The basic idea of phenomenology is making a more strenuous effort to see things as they are, depending on whatever one is focusing on. I think the best scientists and the best artists are precisely that - phenomenologists. We want to know, what it is ... what is it really?

Tenney's curiosity led to considering each composition as an experiment, a process of exploring one or more proposed theories or basic questions of sound and perception. An important influence on Tenney, Cage (1961.2, p. 69) established a precedent twenty-five years earlier in a 1959 article:

What is the nature of an experimental action? It is simply an action the outcome of which is not foreseen. It is therefore very useful if one has decided that sounds are to come into their own, rather than being exploited to express sentiments or ideas of order.

In Kasemets et al. (1984, p. 10), Tenney stated that every composition he wrote "suggests two or three new pieces .... New questions are raised, which a given experiment does not answer, but it does raise the questions, and that's really interesting."

From this perspective his early computer music works of the early 1960s link directly to his later acoustic compositions, as questions raised in the earlier works led to new experiments years later. This experimental process is the characteristic signature of the twentieth-century American approach to composition, and this places Tenney clearly in the Ives, Ruggles, Cage, Partch, Johnston, et al. lineage and tradition. And this process of experimentation opened Tenney to utilize any historical, cultural, and technological influences that he found relevant to a given work, including American folk traditions (indigenous and transplanted), European modernism, and the emerging technology of his time. According to Garland (1984), "Tenney's great creative talent is one of synthesis." As a result his music is eclectic, covering many twentieth-century “isms" without becoming imprisoned by any of them. And throughout his progressive avant-garde work he maintained a conscious respect for and connection with his cultural lineage, to the extent that several compositions were dedicated to other composers.

Tenney interpreted his American experimental heritage as permission to expand beyond the established limits. With no preconceived answers and no preconceived boundaries he was free to experiment openly and freely. This approach established Tenney as the quintessential composertheorist, wherein he posed questions (theories) for experimental study (composition). His resulting aesthetic included the fermenting aspects of experimentation, exploration, liberation, progress, and historical awareness, all balanced with his concerns for practical application and 
human perception. When guided faithfully by these principles a person of integrity really has no choice but to create music and theoretical writings that challenge at least some aspects of the cultural status quo. And James Tenney was a person of high integrity, one determined to avoid the false comfort of any single school or practice, and so he produced work of singular focus and importance. In addition to the elegance and beauty of much of his work, he also influenced several composers and theorists by challenging and encouraging them (us) to consider their own work with the same level of clarity and depth.

\subsection{Form and Structure}

Tenney was singularly concerned with the aural perception of formal structure, the listener's perception of musical change through time. Not restricted to the traditional concerns of tension and release, Tenney proposed a more inclusive theory of musical time. His seminal monographs Meta-Hodos: A phenomenology of $20^{\text {th }}$ century musical materials and an approach to the study of Form, and META Meta-Hodos (1986) and A history of 'consonance' and 'dissonance' (1988) detail his careful thoughts in the areas of formal structure and multi-level perception. As a separate article would be needed to fully present all of the interlocked aspects of these studies, only a severely abbreviated summary is offered here in order to establish some context for the individual compositions examined thereafter.

Tenney defined those formal units of music that can be perceived as temporal gestalt units [TGs]. Specifically, a TG is a unit of composition, existing in time (i.e., with delineated beginning and ending moments), that contains specific statistical specifications of seven primary subjective musical parameters (pitch, loudness, timbre, duration, temporal density, vertical density, and time envelope). TGs have three perceived time references: the time before the TG, the TG itself, and then time after the TG. Statistical specifications are measurable means and ranges for a given parameter. Temporal density describes the number of music units per time unit (which relates to the perceived pace of the music), vertical density is the number of simultaneous events, and the time envelope is the perceived change in timbre over time. Musical form is created by a succession of TGs, and formal shape is recognized (i.e., perceived) through the articulation of structure (the relationship between parts, and between parts and the whole), shape (changes in parameters over time; topology, or contour), and statistical states (the average value and range of each parameter).

Tenney organized TGs on several nested holarchical levels. While many of his early writings used the term hierarchy for these levels of perception, Tenney (personal communication, December 13 \& 14, 1985) subsequently clarified his intentions:

In looking up the etymology of hierarchy, it has to do with orderings of power and value. And I don't perceive what I was calling these hierarchical formal structures as having to do 
with power or value. They're simply hierarchies of inclusion. So, a better term would be holarchy, which means an organization of wholes; an organization of gestalt units.

So, it's not going to actually, literally happen, but I'd like to go back twenty-five years and examine everything I've ever written and locate wherever I've used that word hierarchy; I wish I could replace it with holarchy. I can't now, but you can make this statement for me.

From small- to large-scale, these TGs are labeled element, clang, sequence, segment, section, and the entire work. Successive larger form is perceived by changes in lower-level form; or, conversely, changes at smaller levels generate larger level formal structure. Tenney defined sound elements as the smallest formal level TG. An element may be as simple as a single tone, and it may be a larger aggregate, such as a chord, ornament, or cluster. His most important and significant TG level is the clang, defined as a collection of elements that exists as the primary level of aural perception. A sequence is a cohesive collection of clangs, and segments and sections are progressively larger, yet still intermediate, levels of organization. Tenney's formal definitions relate to Cage's 1949 definitions (Cage, 1961.1, p. 62):

Structure in music is its divisibility into successive parts from phrases to long sections. Form is content, the continuity. Method is the means of controlling the continuity from note to note. The material of music is sound and silence. Integrating these is composing.

For Tenney, the listener's subjective perception is required to create the distinctions between formal TG levels and to also distinguish between events with a given level. While the composer creates the music potential, and the performer brings the music into aural reality, it is the listener who actually interprets the sounds and then creates the resultant structure and relationships. Of course this requires an informed, or at least curious, listener; and this places the listener on equal standing with the composer and performer in the total tripartite equation. A listener can perceive musical continuity within a clang through elements that are presented simultaneously or contiguously, while temporal separation weakens the connection. Depending on the context, simultaneity can create compound smaller clangs, whose elements in turn require further interpretation. Everything in music is delightfully context dependent, and so these distinctions are necessarily fluid. Whatever the level, elements that contain unified or similar parameters strengthen continuity, as does repetition and close variation of specific elements. Within a clang, more intense elements are likely to be perceived as starting points of new clangs or as primary focus moments within their clang. Parameters that change the most serve to define a clang (Tenney called these formative parameters, the determinants of the form), whereas static or more stable parameters serve to define the overall context as well as the start and end times of the clang. The listener's process of perceiving these relationships is further informed by his or her expectations of the immediate future, prompted by the memory of past events in the same music (an objective set) and by referencing other compositions (a subjective set). As with all listening, 
and indeed with all meaningful (i.e., artistic) human activities, the ambiguity increases proportionally with the content and levels of information.

Moving to successively larger levels of perception, and eventually to the work as a whole, formal structure is articulated by the content of lower level TGs. In a recursive process, content creates form for a higher level, and that form in turn becomes the content for the next higher level. Tenney (1971, p. 242) summarized that "it is no longer necessary to treat form and content as fundamentally different things." The largest structural level of composition, the entire work itself, is ultimately delineated in time by the beginning and ending of the music. Tenney grouped compositions into two primary processes at this level. Ergodic compositions are relatively static with statistically homogeneous processes, in which the statistical state of the entire work is equal to the statistical state of each part at the adjacent lower formal level. And nonergodic compositions operate with dynamic processes whose statistical states are not equal across formal levels. These latter compositions include the traditional departure and return structures of the established western tradition (e.g., A B A, sonata, and rondo) as well as A B linear ramp structures. The process mechanism, and the resultant macro formal structure, is ultimately determined by the listener's perception of "same" versus "different" within and across TG levels. So, for the listener, the artistic issue remains focused on two basic questions that need to be continuously asked during the music's performance. If the elements are the same, how are they related? If they are different, what relationships, if any, exist between them?

\subsection{Early Work, Computer Music: Noise Study}

Noise Study was Tenney's first computer music composition completed during his time at Bell Laboratories in New Jersey (1961-64). Polansky (1984, p. 151) noted that Tenney was "the first composer to utilize the music synthesis programs (MUSIC IV) that Max Mathews had been developing." Tenney's music from this period was among the first computer-generated compositions that were more than technical studies or special effects demonstrations. Even with the manifold hardware and software developments during the forty-five years since that time, these early works retain their compositional integrity, intelligent and sophisticated construction, and their direct aural impact and appeal.

With direct access to one of the best computing environments then available, Tenney incorporated the computer into his experiments, both as a tool and as a conceptual paradigm. He wanted to use the computer to explore specific aspects of sound, perception, and his own process of composition. He wrote computer programs to achieve increased variety at micro levels of composition using pseudo-random (specifically stochastic) processes. He was not overly concerned with indeterminacy as a philosophical issue as much as a practical approach to the 
experiment at hand. With a carefully controlled macro structure he allowed the computer processes to work freely at smaller levels. Polansky (1984, p. 151) explained:

The computer provided Tenney with a means to create perceptual domains whose environment he could predict and structure, but whose detail, and even middle level characteristics could in turn structure him, teaching him about his own perceptual processes.

At these smaller levels of recursive holarchical structure, randomly generated statistical means affect smaller-scale events (and means), creating what Polansky (1984, p. 153) termed a "nested gestalt structure." Influenced by Cage, these computer compositions were Tenney's first explorations with removing himself from direct control over certain aspects of the composition process. The computer (with his software subroutines clarifying MUSIC IV) was the tool; the tool informed the experiment; the experiment was the process; the process was the composition.

Tenney noticed that noise and randomness were inherent to all natural processes, whether confined to aural phenomena or expanded to all of physics. He was one of the first artists to recognize and then purposefully utilize this concept. He was interested in understanding and electronically reproducing natural sounding timbres and envelopes (in the context of the early 1960s this was very forward thinking), and he realized that random fluctuations in parameter values led towards this goal. In Noise Study Tenney relied primarily on amplitude modulation $[\mathrm{AM}]$, whereas later works added frequency modulation [FM], including dynamic vibrato. Polansky (1984, p. 158) noted that Tenney later used random AM specifically "to add life to tones." These timbral experiments became a primary focus of computer music much later as software tools advanced; Tenney's work with random fluctuations of timbral parameters predates most of this later activity.

Noise Study was composed in 1961, soon after Tenney began working at Bell Labs. The music is computer generated, but the score is not: later compositions would utilize algorithmic subroutines to generate the score as well (e.g., Four Stochastic Studies, 1962, and Dialogue, 1962-63). Traffic noise, originating from his commute from New York City through the Holland Tunnel to New Jersey, was the early embarkation point for this work. This expanded to noise in general, both literal as sound and as a natural physical process, as the material for this composition. The sound source is a simple AM noise generator with specific initial and terminal values for center frequency, amplitude, and bandwidth. Tenney's program stochastically interpolated parameter values over a given time period between these set starting and ending values. The initial composition combined five such AM instruments, and the final tape assemblage combined three realizations of the initial work at three different speeds, for a total of fifteen computer instruments. The tape speeds included the original realization, plus half-speed and double speed versions. The longest tape duration (half-speed) determined the overall duration of the composition, which is 4.23 . The remaining two realizations were manually 
positioned so that their points of maximum intensity (and maximum temporal density) are aligned with that same moment on the half-speed tape. This occurs at time 2.55 , as shown on Tenney's chart at time 175.1 seconds, (Tenney, 1969, p. 31) [reprinted in Polansky (1984, p. 157)], see Figure 1.
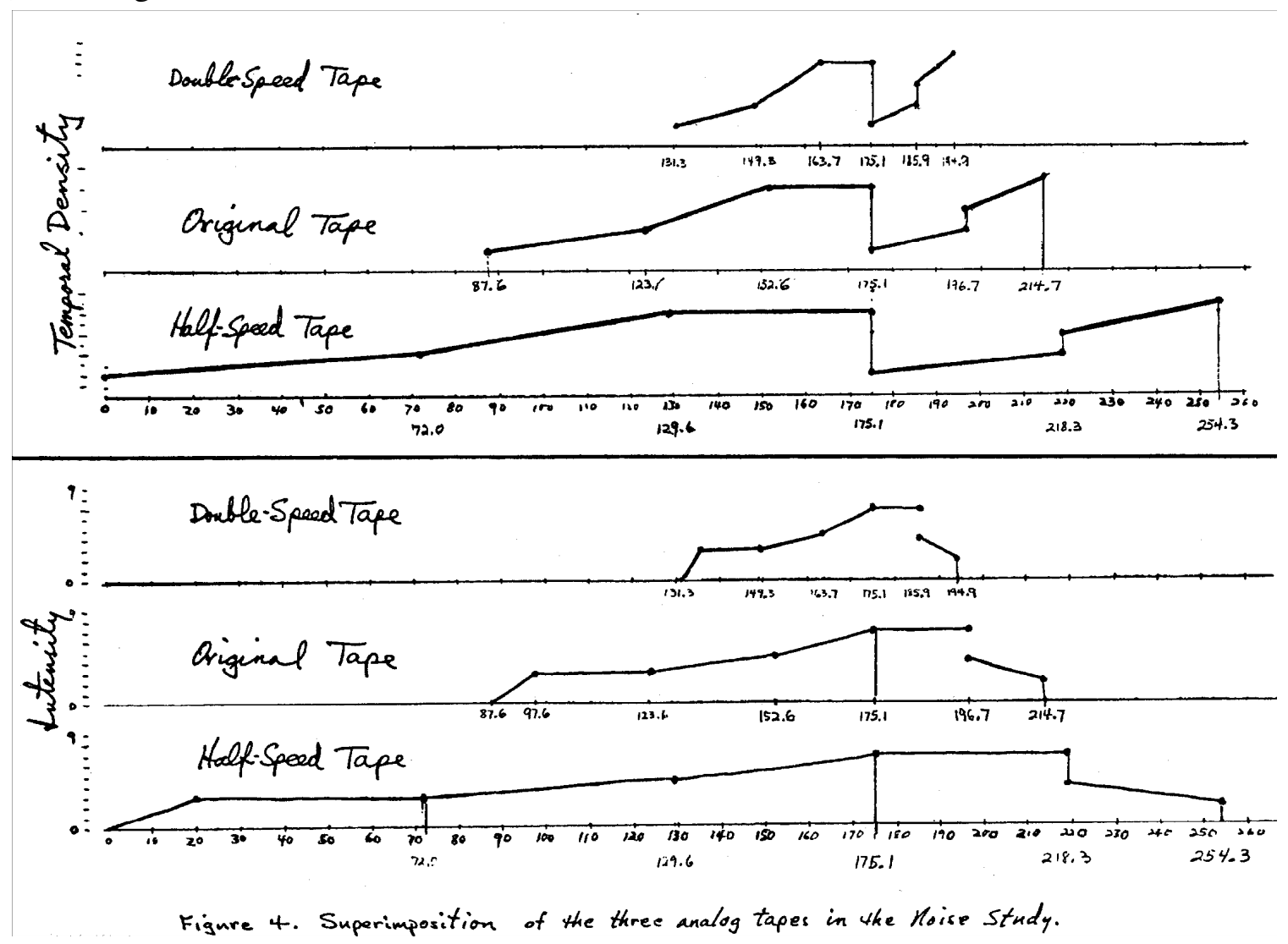

Figure 1. Tenney's Figure 4. Superimposition of the three analog tapes in Noise Study.

This manual superimposition of three manifestations of the same initial Ur-composition and their temporal alignment around the moment of highest density and activity creates a nested recursive structure of clangs at multiple levels. As the second and third tape layers are added and then subtracted in reverse order an overall simple arch form is articulated through sheer density of material. At the same time each tape version proceeds through unified interpolation processes of small-scale exploration within controlled large-scale structures. Established in his early works this dual generative plan remained a favored method for Tenney throughout his composing career.

Noise Study is an early work, of course, and many of the concepts first explored here are more fully developed in later works. Still, the directness of an initial experiment is often more clear, 
simply because the first experiment removes extraneous variables in order to focus on the process and results of the primary experiment. In the midst of this stark exploration of noise, density, and stochastic interpolation, Noise Study remains an engaging work, one that this author regularly programs on concerts in order to introduce audiences to the freshness and timeliness (and timelessness) of this work. Unlike the periodic sine, square, and sawtooth waves that dominated the computer music world at this time, what Goldstein $(1984$, p. 7$)$ described as the "Helmholtzian symmetry that had become the trademark (alas) of synthesized music," Tenney expanded the raw material palate to include noise and other asymmetric waveforms. His interest here was timbre as a primary musical parameter, with noise and randomness used to explore more natural sound fluctuations at the detailed level. Goldstein $(1984$, p. 7) called the result "a rich tapestry of pitch and texture, that set the foundation for a new vocabulary of computer sounds." Noise Study established a solid baseline for Tenney's continued experiments in the areas of timbre, recursive structures, and stochastic parameter details within a controlled macro structure. Later works, both the remaining computer music experiments at Bell Labs and his return to acoustic media afterwards, explored these questions as they raised their own questions for yet further study.

A final note of interest regarding this working period at Bell Labs: Tenney's monograph "Computer music experiences, 1961-1969" was originally titled "Computer music experiments, 1961-1969." This author's typed copy of the pre-published manuscript has the original title with the word "experiments" crossed out and "experiences" amended in Tenney's hand-written block letters. In keeping with the emphasis on experiment as process throughout this article, Tenney's draft version of the title is an interesting insight into his recollections of those early years at Bell Labs.

\subsection{Early Work, Acoustic Music: Music for Player Piano}

Composed in 1964, shortly after leaving Bell Labs, Music for Player Piano is closely linked with the previous computer music experiments. Although performed on an acoustic (albeit machine driven) instrument, the score data for the punched piano roll was computer generated. The algorithmic score was designed to generate increased variety at the small scale while the large scale was predetermined. Only two minutes in duration, the composition was designed to be performed in four different macro permutations, limited by the physical possibilities of running the paper roll through the player piano: forward (P), backwards (R), flipped over (I), and flipped over and backwards (RI). These four performance environments by themselves increase the variety of sonic detail, so that Tenney's interest in parametric variety is here explored on multiple levels of perception. 
Music for Player Piano presents some interesting questions that remain somewhat open-ended. Tenney provided for four performance formats, but what about two, three, or all four versions performed simultaneously? Especially now with the ready availability of digital player pianos this experiment could be mounted. Is this computer music? Is it acoustic music? Is there really a difference? Does the distinction matter when other areas, such as parametric variety and holarchical structural levels, are being explored? A later composition for player piano, Spectral CANON for Conlon Nancarrow, is examined in more detail later in this study.

\subsection{Late 1960s and early 1970s, Postcard music: Beast and Koan}

Collectively referred to his Postcard Pieces, Postal Pieces, or Scorecards, Tenney composed these ten short works between 1965 and 1971. Each score occupied only one page, and they were printed on post cards and mailed to friends and acquaintances. Tenney sent this author a Beast postcard in May 1985 during the early stage of our correspondence, see Figure 2.

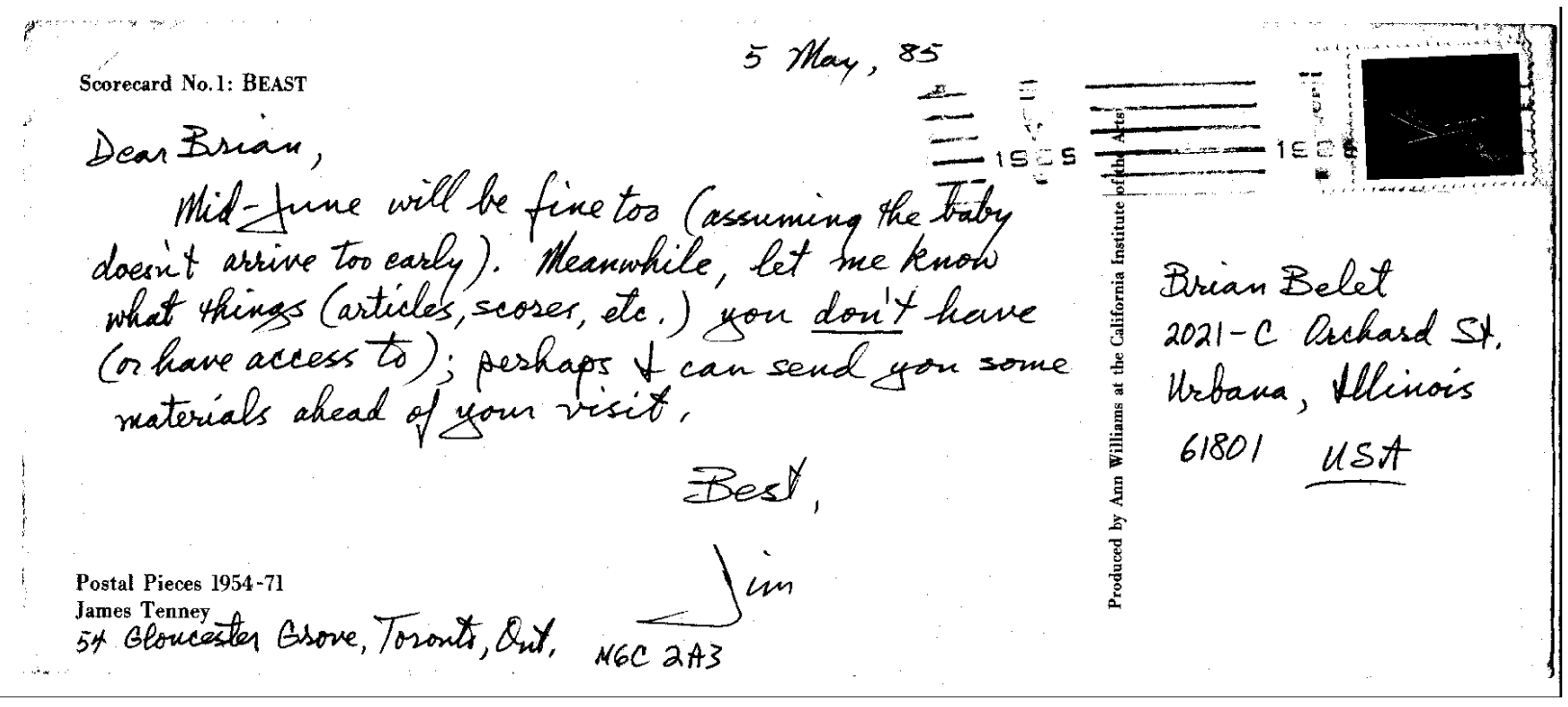

Figure 2. Scorecard No. 1: BEAST Tenney's generosity is evident with his offer of scores and articles in addition to welcoming a visit to his home in Toronto.

For obvious practical reasons each score had to be short and concise, including any necessary performer instructions. The performed music did not have to be either short or simple; only the process needed to be meticulously clear and direct. Most of the works in this federated set address various aspects of intonation, timbre, swell (i.e., the macro arch form), and unifying the structure and the resultant composition as a single gesture. Tenney was interested in articulating a very clear formal structure, with undue drama (at least from himself) removed, so that the 
performer and listener could focus on the unfolding details of the experiment within a single process. These are severely simplified experiments, with unnecessary variables removed (i.e., other parameters left as constant or nearly constant values) so that the questions posed can be fully examined.

All ten miniatures are musical koans, although only one is so titled, and Tenney referred to the full set in this manner. Like its Zen namesake, in which questions are posed, not to be answered, but rather to be pondered endlessly, each composition (Polansky, 1984, p. 196) "asks more questions tha[n] it answers." Goldstein (1984, p. 11) called the set "Haiku for individual musicians." Each fully explores a single process, and the performer is required to enter the process at a microscopic level and articulate the fine details of the process. The process is the performance, which is the composition. The focus areas of the experiments are not new, but here they are clearly focused without the interference of other issues. These works can be included in the overall minimalist grouping as conceptual experiments with singular intents, however they are then ultra-minimalist with their severity of purpose. Other than variations that occur in any live performance these works are completely deterministic, as the notated process is to be followed through to its conclusion. The listener is then invited to simply listen to the change that occurs during this process.

Beast was composed in July 1971 for bassist Buell Neidlinger. The work is a seven-minute study in rhythm, specifically the changes in interval beats produced by slowly changing glissandi double-stops on the double bass. With the A string tuned to $55 \mathrm{cps}$ (its standard tuning three octaves below A-440), the low E string is tuned down to E flat (ca. $38.8 \mathrm{cps}$ ). This open tritone produces approximately sixteen beats per second [bps] (somewhat larger than the just 11/8 tritone of 15 bps actually called for in the score), which approaches the perceptual threshold between a very fast rhythm and a very low resultant difference tone. From this extreme rate boundary the music establishes a stable rhythm of zero beats with a unison A1. Notated on graph paper the score contains target values for beat frequencies connected graphically by a series of sine wave performance curves, with primary target crests supported by nested smaller crests, see Figure 3. 


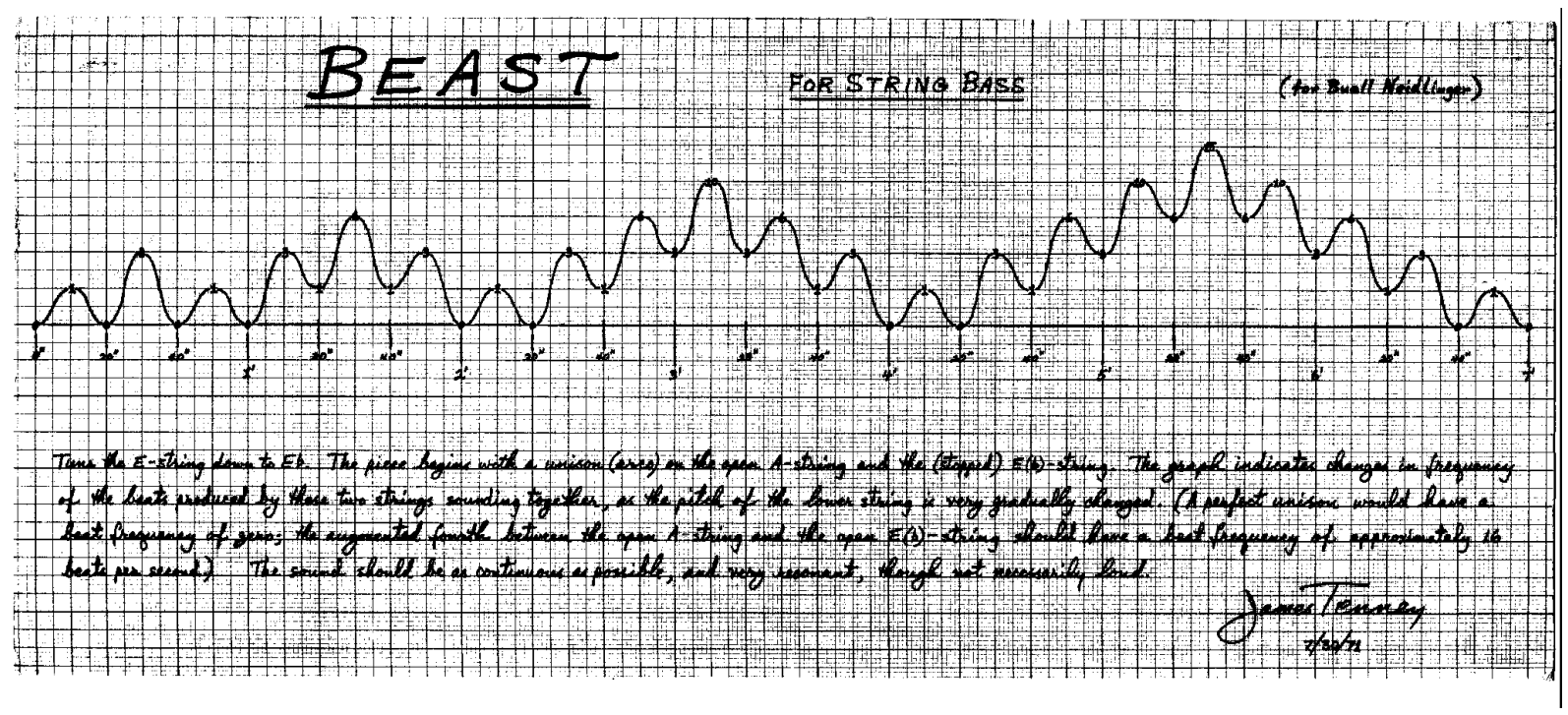

Figure 3. Tenney's score for Beast, printed on a postcard sent to this author, and also reprinted in Polansky (1984, p. 197).

Dated "7/30/71" Tenney's hand-written and signed score carries the following performance instructions:

Tune the E-string down to Eb. The piece begins with a unison (arco) on the open A-string and the (stopped) E(b)-string. The graph indicates changes in frequency of the beats produced by these two strings sounding together, as the pitch of the lower string is very gradually changed. (A perfect unison would have a beat frequency of zero; the augmented fourth between the open A-string and the open $E(b)$-string should have a beat frequency of approximately 16 beats per second.) The sound should be as continuous as possible, and very resonant, though not necessarily loud.

The formal structure, articulated by the frequency curves by number and relative beat intensity targets, is recursive and the timing of the expanding swells aligns with the Fibonacci series $(1: 1: 2: 3)$. The primary target crests are three bps at 0.30 (midway through the first one-minute swell), six bps at 1.30 (midway through the second one-minute swell), ten bps at 3.10 (within the third swell, with duration of two minutes), and fifteen bps at 5.30 (within the final three-minute swell). The nested interior swells within each primary swell are organized as palindromes, with bps targets of $1,3,6,10$, and 15 approaching and retreating from each respective primary target. The approximate intervals created by these beat targets are a 55/54 sixth-tone for $1 \mathrm{bps}$, a 55/52 flat half-tone for $3 \mathrm{bps}$, a 55/49 flat whole-tone for $6 \mathrm{bps}$, a 55/45 (11/9) neutral third for $10 \mathrm{bps,}$ and a 55/40 (11/8) just tritone for 15 bps. 
Koan, for solo violin, was composed in August 1971, the month following Beast. Koan was composed for Malcolm Goldstein, violinist and composer, and the work was later expanded into Koan for String Quartet, which is examined briefly later in this study. Like Beast, Koan is a detailed study in solo string double-stops. Here the double-stops are performed as constant tremolo alternations, and the ever rising interval changes evoke the rising minor sixth Shepard tone gestures of his electronic work For Ann (rising) from 1969. The score indicates that the violinist begins on the open G-D just perfect fifth, with the G slowly ascending until it reaches the unison D4; the ascent continues on that same string until the next perfect fifth is reached with A4. At this point the performer dovetails to the open D-A combination and continues the process creating the aural illusion of perpetual ascending motion. The end point passes through the E5B5 perfect fifth with a decrescendo to a final E5-E6 octave. Tenney's performance note is brief and placed under the final decrescendo gesture: "gradually move toward bridge, until nothing but noise is heard," see Figure 4.

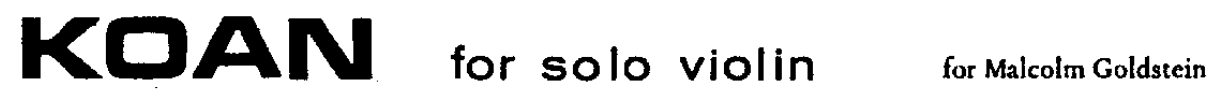

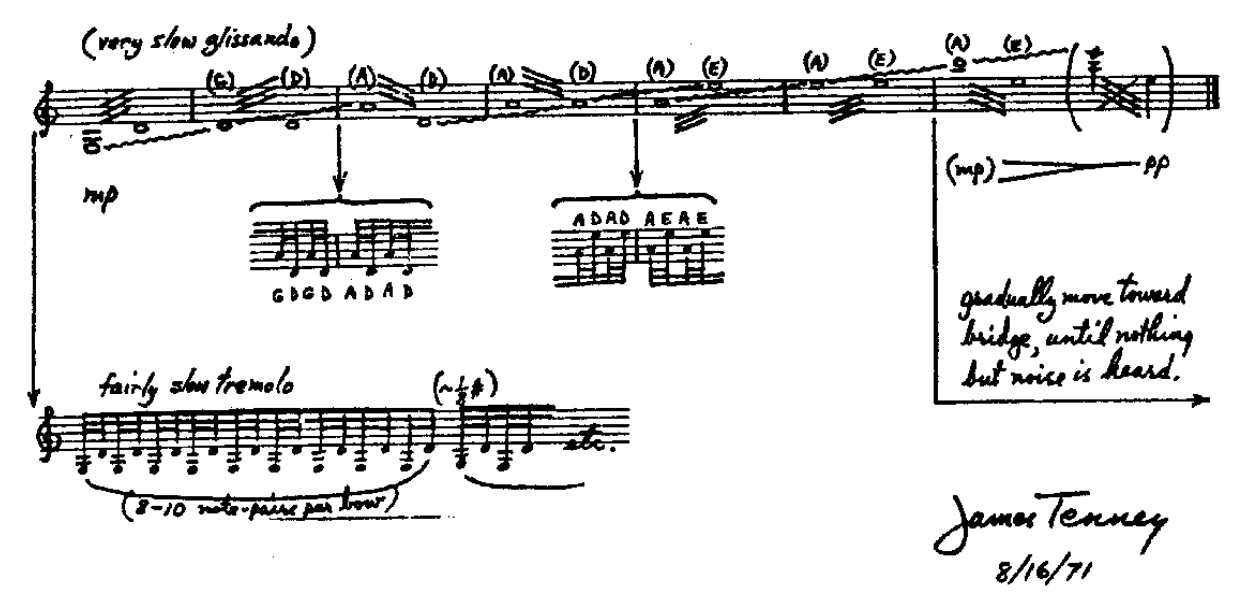

Figure 4. Tenney's score for Koan, reprinted in Polansky (1984, p. 200).

The performance time for Koan is not indicated, and so it can be quite long. Goldstein (1984, p. 11), reflected on his performance of this work: “The 'Koan' for my solo violin - I still approach and learn what it is to allow a graceful motion of the arm without thought and to hear the violin shimmering in space." 
These two miniatures are literal and detailed explorations into the interrelated workings of intonation, timbre, harmony, and rhythm. Unfretted, bowed strings are the ideal media for these experiments on the continuum that contains these parameters. With the composer's personal drama removed from the process, that burden falls to the performer. Even though the works are objective experiments, in performance they can be transformed into dramatic journeys of their own, journeys that require careful listening of the gradually unfolding changes.

\subsection{0s: Spectral CANON for Conlon Nancarrow}

Composed in 1974 for retuned player piano, Spectral CANON for Conlon Nancarrow is a largescale physical manifestation of a very simple harmonic experiment. With the player piano tuned justly to an A harmonic series (with low A1 as the fundamental tuned to $55 \mathrm{cps}$ ) Tenney proposed to literally map the harmonic ratios of each interval to duration and temporal structure. This may be the first musical example of sonification, which is now seriously researched in science and industry. While this is very simple in concept, it presents a complex mathematical problem to solve, especially when he added independent accelerando and decelerando for each voice to the equation. With a need for absolute precision (since that is why a composer would select a mechanical player piano as the performance medium) Tenney (personal communication, December $13 \& 14,1985$ ) wrote a computer program to calculate the ever changing proportional attack times for each voice: "I used it to make sure that the note durations would be absolutely precise. But I didn't need the computer program to tell me anything else about the piece."

The full piano range is used to permit the overtones to be used in their naturally occurring octaves with respect to the low fundamental, with the highest note E6 articulating the $24^{\text {th }}$ partial. Tenney termed the just-tuned instrument a "harmonic player piano," both to reflect its new tuning and to highlight the focus of this experiment. The attack time score data was transcribed to a paper piano roll and actually punched by Nancarrow on his unique machine in Mexico. Tenney composed a 24-voice proportional rhythmic (temporal) canon. The fundamental A1 enters first with a four second interval between successive attacks (duration), and immediately begins to slowly accelerate. This four-second interval was an arbitrary choice for constant value ' $\mathrm{k}$ ' in the computer program. When the fundamental's duration has decreased to two seconds, the second voice (second partial A2) enters also with an initial duration of four seconds followed by its gradual accelerando. As the compound accelerando continues these two voices remain in a strict 2:1 rhythmic relationship to reflect their octave harmonic relationship. The third voice (E3) enters next with a temporal relationship of 3:1 to the fundamental (and 3:2 to the second voice), and this process continues as each successive voice is added in turn. The target for each voice in the accelerando process is 192 iterations. When the first voice reaches this target it begins to gradually decelerate for another 192 iterations. This midpoint of the fundamental's palindrome aligns with the entrance and accelerando of the final voice. This point is also an attack 
simultaneity for all 24 voices, which marks a moment of highest vertical density. The process ends at another point of complete synchronicity when the fundamental has completed its decelerando and the highest voice has completed only its accelerando, and each of the interior voices is at some proportional point along its own path.

For this work Tenney constructed a very precise small-scale plan for the unfolding structure of each individual voice using recursive mathematical calculations. The overall macro structure, which takes slightly longer than three and a half minutes to unfold, is a direct consequence of his detailed micro plan. Like his other experiments, Tenney set up the conditions and the process, and then let the music unfold according to that plan. The resulting sound is an ever-increasing wedge of sound, in terms of number of notes, width of register, rhythmic complexity, and composite timbre. As the fundamental and lower overtones decelerate near the end of the process, there is a simplifying of rhythm on that level that is counteracted by the still increasing energy in the higher overtones. The final moments present a cascade of harmonic timbral sweeps that literally sound the way the score looks, see Figure 5.

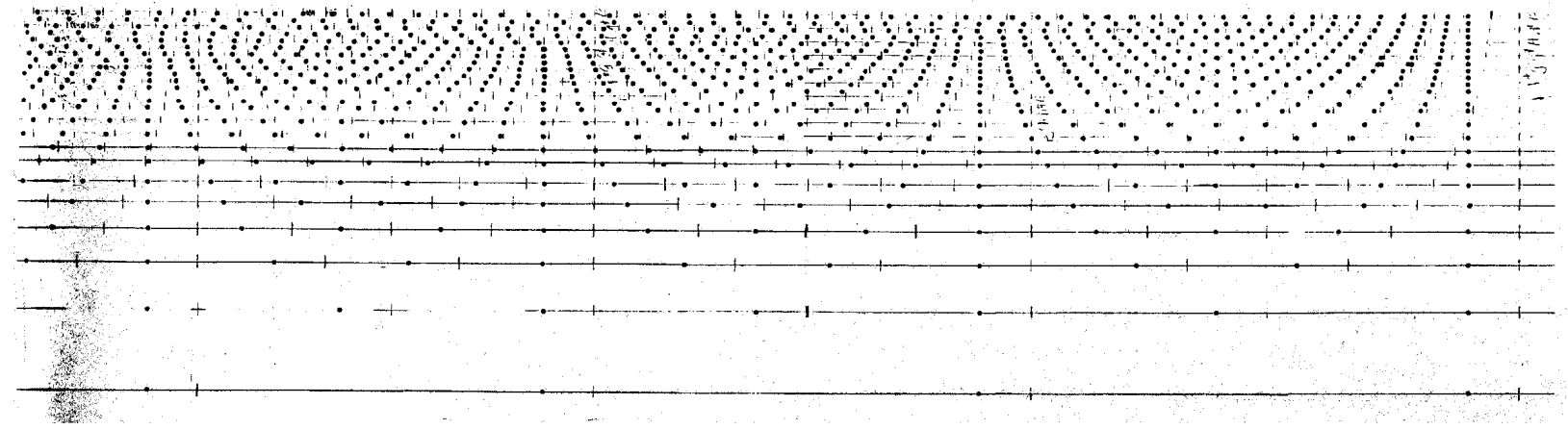

Figure 5. The final page of Spectral CANON..., compressed and reprinted in Musicworks 27, p. 17.

As Polansky (1984, p. 225) observed, this concluding area presents "some breathtaking 'parabolas and hyperbolas' [which are] a natural result of the logarithmic cross rhythms." The final cadence is an abrupt truncation of the process, and only the fundamental first voice completes the dual process of accelerando and decelerando. While the actual result is literally asymmetrical due to this truncation, the process is easily perceived as balanced and symmetrical, largely because the listener can readily track the lowest voice, which is complete, and the highest voice, which completes it first half of the process. 


\subsection{0s: Three Indigenous Songs}

Three Indigenous Songs is one of Tenney's most curious, and ultimately fascinating, timbral experiments. His thesis was to create a timbral analog for the human voice using acoustic instruments. In perhaps the most extreme implementation of his basic question, "What would happen if ...," this collection from 1979 uses three pre-existing songs (including his own 1971 setting of translated Iroquois chants for the final song) as literal source material for his timbral transformation in these extreme Klangfarbenmelodie textures.

The ensemble includes two piccolos, flute, bassoon (or tuba), and multiple non-pitched wood and metal percussion. According to Polansky (1984, p. 251) these instruments were not used to imitate the human voice, but rather to "simulate the various formants and noise transients of the different speech sounds." In this way Tenney synthesized the human voice by means of instrumental approximations, an abstracted process that allowed him to experiment with timbre and formal perception. He followed a strict and literal transcription of recordings of the three songs, which results in another microscopic study of timbral detail, in this case the details of vocal acoustics. The percussion battery was used to articulate vocal consonants (fricatives and plosives). The woodblock was assigned to the sounds produced by the letters $\mathrm{k}, \mathrm{t}$, and $\mathrm{p}$; the tomtoms simulated $\mathrm{g}, \mathrm{d}$, and $\mathrm{b}$; the wire brush handled th, $\mathrm{f}$, and $\mathrm{h}$; and the suspended cymbals were used for s and sh. Vowel harmonics were assigned to the piccolo and flute, and the vowel fundamentals were performed by the bassoon (or tuba). Each of these instruments has a relatively simple spectrum, and Tenney kept each instrument in an isolated formant region. The result is once again experimental clarity with controlled variables.

Each song has a distinctly American text and setting. "No More Good Water" is a 1920s recording of slow vocal blues with harmonica performed by Jaybird Coleman. Tenney recorded himself reciting Walt Whitman's “Kosmos” for the second song. And Tenney's 1971 choral setting of "Hey When I Sing These Four Songs Hey Look What Happens" is the final song. This is an eclectic collection, which fits into Tenney's overall aesthetic. The blues song is rooted within the African-American heritage, Whitman's poem and Tenney's reading both reflect European transplanted culture, and the Iroquois healing chant transcription calls upon our First Nation elder heritage. Tenney maintained a conscious connection with his American culture, contemporary and folk. Rather than quote folk tunes or employ folk idioms, he preferred to evoke the general influence of the culture at an underlying level. In doing so he portrayed a genuine American sound without resorting to the clichés of Americana.

In "No More Good Water" Tenney used the flutes to simulate the antiphonal harmonica interludes that occur between the sung lines. Tenney transcribed his own speech rhythms and timbral inflections for his reading of "Kosmos." For "Hey When I Sing ..." he retained the composite rhythm of his original SATB setting, and the soprano solo was transcribed into 
instrumental interludes between each line. The last song is a twice-removed transcription, since his 1971 choral work was a setting of a translation of collected Iroquois chants, and this 1979 instrumental version was a literal transcription of that SATB setting. Once established, the instrumental transcription process was strictly enforced. This was necessary in order to allow the experiment to produce reliable results. The results of this experiment, as this author reported in an earlier study (Belet, 1990, p. 37), created "a composition that is directly determined by the acoustics of the human voice rather than an accurate instrumental imitation of the voice." In Three Indigenous Songs the formal study and resulting artistic expression are one and the same. With the strict transcription process, the intellectual control and emotional strength of the original material are unified and very difficult to separate. The aural result creates a higher gestalt experience than either aspect provides alone.

\subsubsection{0s, Glissade.}

Glissade is a five-movement work for viola, cello, double bass, and analog tape-delay system, composed in 1982. Each movement is a separate deterministic process composition, and the string instruments permit further experiments in intonation as well as provide a homogeneous ensemble of similar timbres. The tape-delay system enhances the overall string texture by smoothing the boundaries of temporal change. The delay time is set at five seconds with a large decay of thirty seconds. Tenney used a tempered tuning system as a practical close approximation of extended just intonation. In an equally practical mindset he devised a notation system of up and down multi-head arrows to indicate specific pitches. Glissade straddles two compositional periods for Tenney: the first four movements refer to his preceding harmonic series period, whereas the final movement foreshadows his following period that deals with lattice pitch-space fields.

Using seventh-tone micro divisions of the semitone, Tenney divided the octave into 84 tempered pitches, when arranged in scalar order. This provides a close workable approximation of extended just intonation up through the $32^{\text {nd }}$ partial. Tenney's use of small intervals here was to provide a practical performance approach to extended just intonation, and was not intended to be a microtonal field or theory. His multi-head arrows were also a practical solution for performance, in which up to three arrowheads up and down, plus a center pitch without an arrow, created a clear way to notate each seventh-tone. He kept the viola and cello in standard just tuning ( $\mathrm{C} \mathrm{G} \mathrm{D} \mathrm{A)} \mathrm{and} \mathrm{retuned} \mathrm{the} \mathrm{bass} \mathrm{as} \mathrm{needed} \mathrm{for} \mathrm{different} \mathrm{movements.} \mathrm{All} \mathrm{strings} \mathrm{were}$ aurally tuned to beatless just open fourth and fifth intervals. Tenney accurately observed that string performers intuitively attempt to aurally tune intervals via just ratios, and so he used his seventh-tone theory as a working outline with the understanding that the performers would actually tune to the referenced just intervals. This is easier to accomplish in slow tempi and thinner textures, and the first and fourth movements permit this to a high degree. A detailed 
analysis of Glissade is presented in Belet (1990), a study that is still readily available (in contrast to the earlier Soundings 13 and Musicworks 27 sources which are now difficult to obtain). Only a brief overview of each movement is presented here to further illustrate Tenney's unity of formal structure.

The first movement, "Shimmer," is a harmonic glissando canon using only open node string harmonics. Once all three instruments have entered (viola, cello, then bass) a composite texture of constantly changing pitch and harmonic relationships emerges from the glissandi process. Perceived large-scale harmonic sections are alternately heard as stable (C harmony), unstable (transition), stable (A harmony), unstable (transition), and finally stable (return to $\mathrm{C}$ harmony). With duration of 8.10 (including the thirty second tape decay), these sections temporally interrelate with a 3:2:1:2:2 balanced proportion. These sections are actually perceived more through texture changes, as the long durations hinder hearing the direct time relationships. While the small-scale pitch centers ascend by fifths (C-G-D-A) the large-scale perceived harmonic shift through the stable areas from $\mathrm{C}$ down to $\mathrm{A}$ is a minor third relationship. The direct perfect fifth relationships are obscured by their placement within the multi-harmonic transition sections. This perceptual ambiguity among the structural harmonic levels adds to the music's allure, and the dilemma is not addressed (thankfully). The transitions contain several polyphonic linear glissandi clangs with a variety of pitch anchors, whereas the more stable harmonic areas contain drone clangs (extremely stable) and glissandi, which are anchored within the harmony. The final section settles eventually on a stable C E G just triad, and the tape delay prolongs this sonority into the whisper ending. The movement is isomorphic with a macro arch shape. Pitch is clearly used as the formative parameter, as it changes more rapidly than any other parameter. Overall cohesion is achieved by constant timbre and envelope (including loudness), and is further strengthened by very slowly changing vertical and temporal densities, which change from low to high during the glissandi.

"Array (a'rising)" is another canon. As the title implies, this second movement relates to Tenney's earlier For Ann (rising) (1969). The voices enter in turn, with a three-beat time interval, with rising glissandi producing a quasi-Shepard tone texture. The full Shepard effect is not achieved with only three voices, yet the clarity of the trio permits the canonic process to be readily perceived in performance. With duration of 5.45 the macro structure is a single large compound resonant clang with an arch shape (delineated by pitch targets) and a final register ascent. Dynamic swells and the tape delay mask interior divisions that would otherwise articulate smaller clang divisions. Like the first movement pitch is the formative parameter while the remaining parameters define the context. The pitch plan for each voice ascends through a C E G\# augmented triad two and a half times before reversing course down to the initial $\mathrm{C}$. The final ascent aims for a high $\mathrm{C}$ and then continues up with a fade out and tape delay. 
"Bessel functions of the first kind" is a shorter gestural canon, with duration of 2.40. Unlike the first two, this movement calls for an abrupt cut off of the tape delay system when the three strings complete their notated processes. In this multi-movement performance context this abrupt ending is a very dramatic gesture, and it remains one of this author's favorite works from this time period. Beginning with high and low pitch boundaries each instrument aims for the eventual target pitch of $\mathrm{A} 3$ following glissandi curves articulated by Bessel functions of the first kind (see Belet, 1990, pp. 52-54 for a more detailed examination of this process). These Bessel function curves create an ever-narrowing pitch range, which result in the pitch trajectories drawn in Figure 6.

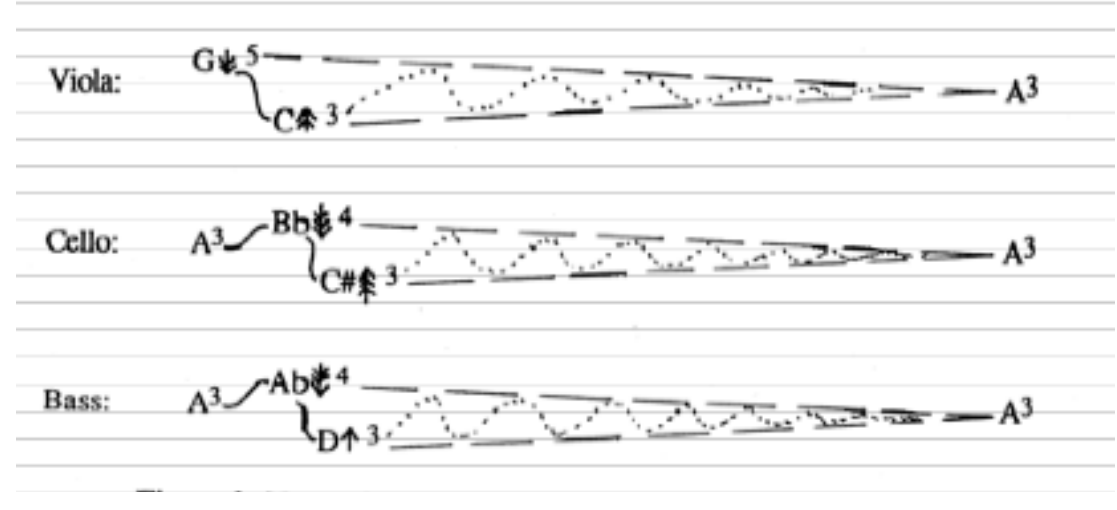

Figure 6. Narrowing ranges (delineated by Bessel function curves) in "Bessel functions of the first kind."

The fourth movement, "Trias harmonica," is another canonic process. A single gesture occupies seven minutes, and the tape delay system strengthens the single resonant clang structure. This was Tenney's favorite movement of the set (personal communication, December $13 \& 14,1985$ ), and it utilized what he called "plastic glissandi." The bass holds a D3 harmonic throughout the movement, and both the viola and cello reinforce this same pitch in staggered canonic entrances. The viola gradually ascends linearly to A3, while the cello descends exponentially to D2, as shown in Figure 7. 


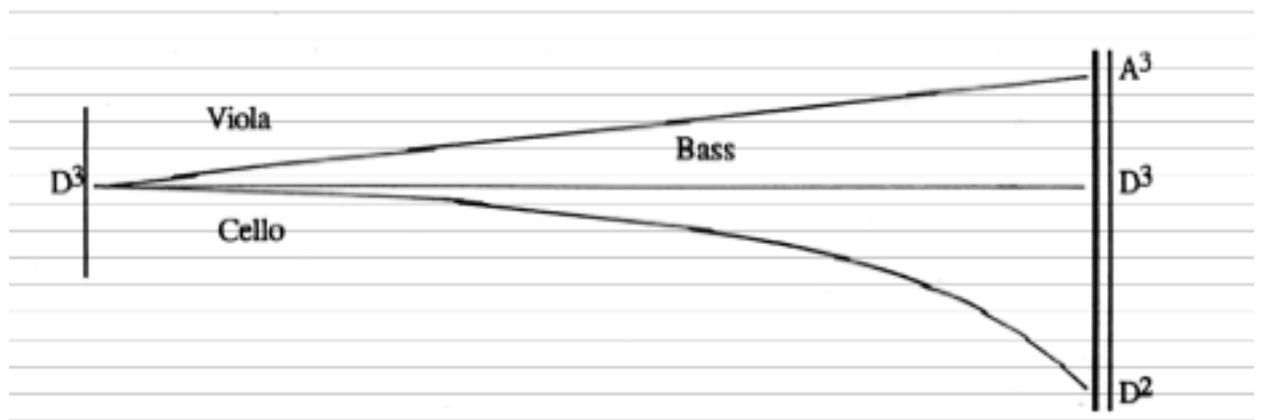

Figure 7. Pitch contours for "Trias harmonica" showing the viola's linear ascent and the cello's exponential descent with respect to the bass' reference drone.

Throughout this process the interval between the cello and the bass is always the next proportional interval down in some harmonic series with respect to the interval between the viola and the bass. That is, the bass drone D3 is always the harmonic mean of the two outer expanding pitches. Tenney (personal communication, December $13 \& 14,1985$ ) described this relationship as maintaining proportionally related intervals in some harmonic series, which itself keeps shifting: "The difference will always be the same. [And,] there are an infinite number of harmonic triads." The score indicates only a few specified target intervals and ratio relationships at certain performance time points. The perpetual glissandi in the viola and cello automatically cover the intervening interval relationships, provided the performers shift their pitches slowing while listening closely to the emerging harmonies. The macro structure is an expanding ramp shape, with slowly changing pitch and duration used as the formative parameters. The very careful exploration of harmonic space in this movement relates to Tenney's next composition, Koan for String Quartet.

The final movement is the longest, at eleven minutes, and is titled "Stochastic-canonic variations," through which Tenney returns to stochastic processes for detailed pitch determination. This movement simultaneously refers to the first four movements as another experimental process composition, and it also presages his next group of compositions. This movement is a canon in texture and style, with the specific pitch details determined stochastically. Within the overall canon Tenney structured an introduction and a set of variations. The actual stochastic canon process establishes a single pitch contour with a unified statistical evolution for each instrument within each variation, while the specifics of pitch detail differ. The resultant pitches of the Introduction are subsequently reduced in each successive variation by means of a stochastic subtractive sampling process. Each new variation therefore contains less specific information than the previous statement of the music, both in terms of actual pitch numbers and the octave and fifth octave duplications, see Figure 8 . 


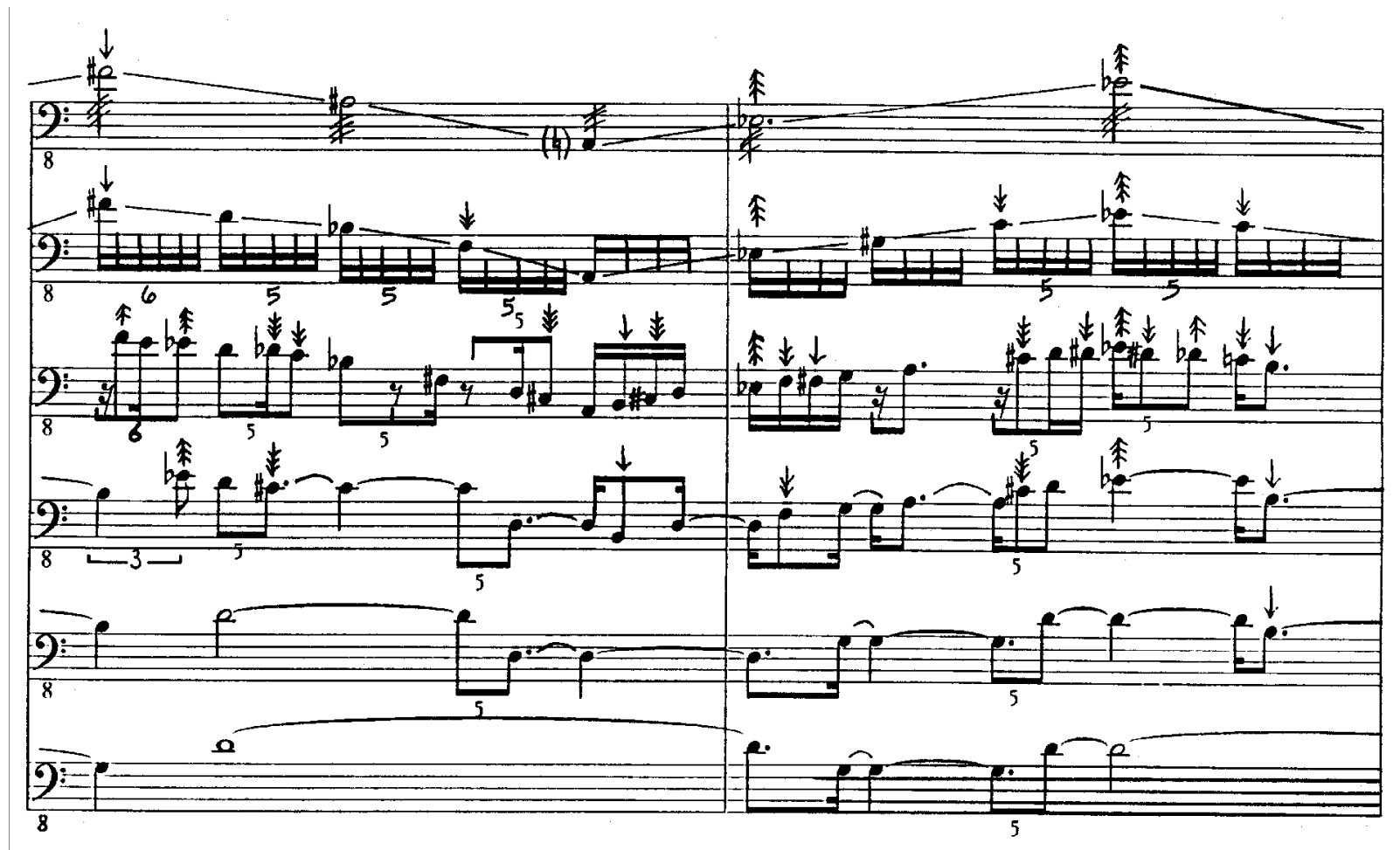

Figure 8. Two-measure excerpt of superimposed bass music from "Stochastic-canonic variations" showing Tenney's subtractive sampling process. The top line is from the Introduction, with Variation 1 below it, and continuing down to Variation 5.

This reduction process generates results that are progressively more deterministic, which is an interesting goal within a stochastic process. As Tenney (personal communication, December 13 $\& 14,1985)$ said: "It was in the nature of that piece that once I have one section worked out (according to the process that I have designed) then everything else came from that - in a sense, predetermined." This directed pseudo-random process relates to Tenney's later compositions Bridge and Changes.

Primary articulation characteristics, including dynamic levels, identify each section and therefore delineate changes between them. This articulation plan, the context setting parameter within each variation, is summarized as:

$\begin{array}{ll}\text { Introduction } & \text { arco tremolo, fff } \\ \text { Variation } 1 & \text { arco tremolo, fff } \\ \text { Variation } 2 & \text { col legno battuto, ff } \\ \text { Variation } 3 & \text { pizzicato, } \mathrm{f}\end{array}$


Variation 4

Variation 5 legato (arco), $\mathrm{mf}$

sustained (arco), thinner texture, $\mathrm{mp}$ to $\mathrm{p}$

The duration of each section is largely balanced: the Introduction includes each instruments' first 60 beats (at a tempo of quarter note $=60 \mathrm{bpm}$, this equates to 60 seconds); Variations $1-4$ are each 90 beats; the first part of Variation 5 (mp dynamic level) is also 90 beats, followed by an additional 61 beats at the lower $p$ dynamic level, with even fewer pitches in a dissipating texture. This author actually hears Variation 5 as two separate sections, and Tenney even emphasized this division with a double bar in the score after the first 90 beats. Following Tenney's thesis that the listener's perception is key to the structure of the music, this author will identify this final variation as Variation 5a and Variation 5b, respectively, and will consider them as two separate sectional clangs. For each (primary) variation the voices enter canonically with a time interval of 30 beats in the set order bass, then cello, then viola.

The details in the Introduction and Variation 1 are difficult to hear due to the aggressive articulation context. Gestures within successive variations are progressively easier to perceive as the articulations calm down and the density of material thins out. Variation $5 b$ is essentially a Coda, and its truncated length and fade out gesture create a convincing ending to this movement. This work displays a metamorphic structure, as the variations' clangs all relate to each other through the subtractive process. The macro shape is both an arch (with the Introduction presenting the pitch range and curves, and Variation $5 \mathrm{~b}$ anchoring the end as a Coda) and a contracting wedge (through decreasing density). Pitch is the most active formative parameter, with loudness, timbre, and temporal density changing at the section (variation) level,

All five movements of Glissade are focused harmonic experiments that utilize canon, glissando, with a swell (arch) shape. Each is a single large process, koans that raise more questions about harmony and compositional technique than they answer. As such they invite the listener to critically reconsider the working definition of harmony and its role in music (function, decoration, or something else). Tenney retains his signature economy of means, yet still creates a large variety within the timbre and density parameters.

\subsubsection{0s, Koan for String Quartet}

Tenney composed Koan for String Quartet in 1984 to address some perception questions that remained from his 1971 Koan, for solo violin. Without a sounding reference fundamental tone, the double-stop glissando upper partials of the solo violin can have multiple interpretations, whereas the string quartet provides that reference with drones. The solo violin music of the earlier work is essentially the Violin 1 part for the ensemble, but notated here with more specific pitch targets and a more precise rhythm. These practical changes were made to integrate and 
synchronize with the other three instruments. The ensemble has a somewhat different ending gesture, and the overall duration is controlled at approximately twenty minutes. Koan for String Quartet is Tenney's second run through this experiment, his attempt to explore intonation, harmony, and timbre with more precision and clarity. Like it predecessor pitch is the primary formative parameter, supported by loudness, density, and the overall arch shape. Timbre, duration, and envelope are the more constant contextual parameters.

Informed by Glissade, especially the fourth movement, Tenney again explores harmonic space implied by the harmonic series using tempered close approximations of extended just intonation. The reference drones provided by the three lower strings change throughout the process, assuming a variety of functions (sometimes the fundamental, sometimes other related partials). Unlike both earlier referential works, Koan for String Quartet contains interior resonant clangs that are delineated primarily by the entrance and harmonic shift of the drone voices. Tenney here used sixth-tones for his just intonation approximations as they most nearly aligned with his target simplest just ratios. The score is fully annotated with both cents deviations from the tempered notes and the target just ratios, see Figure 9. 

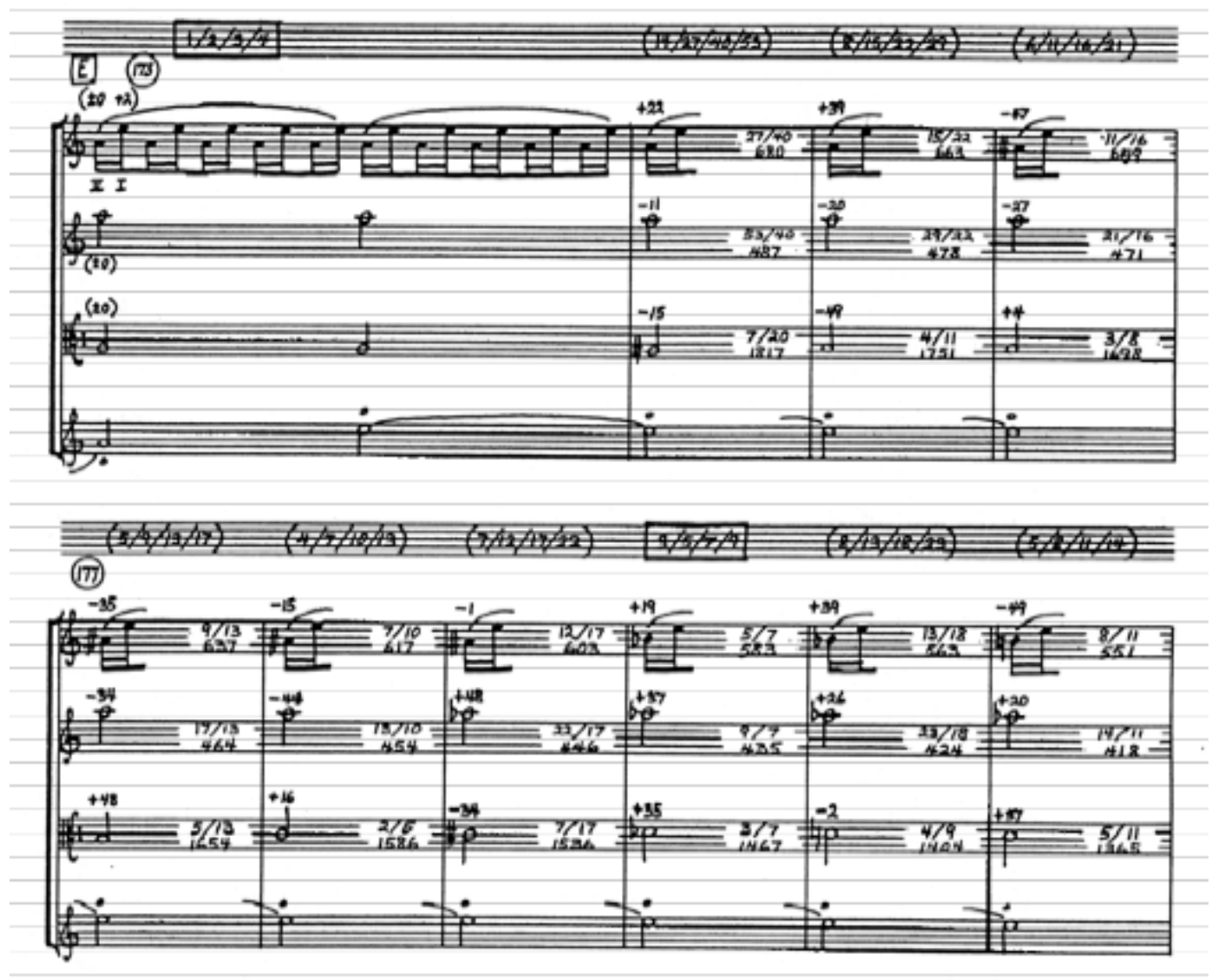

Figure 9. Score excerpt from Koan for String Quartet showing Tenney's cents and ratio annotations.

Tenney (personal communication, December $13 \& 14,1985$ ) understood the visual impact of this score: "This is utterly precise in its notation. It's very intimidating from the players' approach until they understand that they can analyze and actually learn to hear the harmonic relationships." Many of the target harmonies are easily heard stable sonorities. Once mastered, the first violin gradually interpolates between these points through glissando. The second violin and viola articulate specific difference tones relating to the first violin double-stops, and the cello doubles the fixed drone of the first violin for most of the composition. The cello adds additional resultant tones in the final two sections, thus increasing the harmonic complexity through a Coda section. Timbre, durations, and loudness also change more significantly during this final section, strengthening the sense of conclusion. The overall single process is preserved from the original Koan and is also redefined with seven sectional clangs (see Belet, 1990 for a detailed analysis). The process, the experiment, is easy to perceive in performance, yet remains very difficult to perform. It remains a koan. 


\section{Conclusion}

James Tenney was well versed in mathematics, science, poetics, politics, as well as music. All of these disciplines converged to inform his work as a composer and as a theorist. In his pursuit to understand sound and its relationships to our existence, he placed himself (knowingly or not) in a position of social leadership. Garland (1984) summarized this well: "Thus he is aware of the deeper responsibilities of a radical artist in today's society. A commitment to a kind of creative truth that transcends the mere technicalities of the work." When asked by this author to elaborate on his role as a composer in society (Belet, 1987, p. 460), Tenney returned to his central concept of curiosity:

If I'm satisfying my own curiosity by making some music of a certain kind, then it seems likely to me that there will be others whose curiosity is also satisfied by the music. Now, maybe curiosity is too simple, but I'm using it as a term to cover a lot of things there. Whatever it is; that need to know, that need to understand, that need to perceive more of these ideas .... And there will be others that want that.

Throughout his varied output as a composer, from early computer music to subsequent acoustic media, from miniature postcards to large-scale extended works, from solo performer to large ensemble, Tenney utilized consistent tools and concepts to maintain compositional unity and formal clarity. Fueled by curiosity and working within an eclectic aesthetic rooted in experimentation, Tenney favored an economy of ideas and materials within a given work (often severely limited to a single focus) and an avoidance of undue drama. He usually relied on a pronounced clarity of orchestration, and often employed monothematic structures to direct the listener to focus on other parameters as the focus of the experiment. This simplistic approach generated synergetic works, often with a high degree of aural complexity.

For Tenney the clang was the primary sound unit, with its own parameter characteristics operating on multiple levels of aural perception. He favored ergodic over dynamic processes, and many of his works rely on canonic motors to drive them forward. A large-scale arch form was often used to create a macro sense of temporal arrival or conclusion. Does this form shift an ergodic process to a dynamic one of departure and return? While not Tenney's intention, such a structure imposed at the largest level of time can create such an imaginary journey, and it certainly does provide an inner sense of arrival at the end of the composition.

Many of his works are metaphorically offered as Zen koans. Maybe all of his compositions are koans - the more this author listens to Tenney's music, the more they circle around and around posing more questions to contemplate while still somehow making sense without words to analyze them. They are interesting questions, without necessarily providing any "correct" answers. Not just circular, Tenney's overall process was (and is) a non-ending connected spiral 
of theory, composition, evaluation, theory, composition, evaluation, theory .... These aspects coalesce into a lifelong unity of purpose, resulting in a macro formal continuity that contains all of his theoretical writing and music compositions.

Throughout his life Tenney maintained his individuality, his rigorous depth balanced with a wry sense of humor. He was influenced by many composers, and he generously gave them credit in his writings and through the subtitles and dedications of his compositions. Yet, only he sounded like James Tenney, and his music never sounded like those who influenced him. His music explored and then surpassed the various -isms of his day, and then he moved on to other experiments. Always other questions beckoned and his work moved in whatever direction he needed to pursue. This willingness to follow the path he needed to follow, to remain true to his own aesthetic, to not be distracted by popular whims (even those more esoteric whims that disrupt academia and other so-called bastions of serious art music), and his generosity with younger composers searching for their own paths are James Tenney's lasting legacy as the twenty-first century settles into its cultural stride. He music and writing will be studied and analyzed for many years, and the current generation of composers has a large debt to happily repay.

\section{Acknowledgements}

Research and writing time for this article was supported by the College of Humanities and the Arts, San Jose State University, through a release-time grant during the Spring 2007 semester. Larry Polansky, perhaps the leading champion of Tenney's music and writings for many years, generously provided scores and monographs before they were published when this author began studying Tenney's music during the 1980s. Larry has remained just as generous with his knowledge and encouragement during the intervening twenty years. And special thanks go to Marianne Bickett, my wife and partner, for reading drafts of this paper and offering useful suggestions throughout the process, and for understanding and supporting the long hours needed to pull the various threads together.

\section{References}

Belet. B. (1987). An interview with James Tenney. Perspectives of New Music, 25(1\&2), 459466.

Belet, B. (1990). An examination of the theories and compositions of James Tenney, 1982-1985. Dissertation Abstracts International, 51(04).

Budd, H. (1987). [no title] Perspectives of New Music, 25(1\&2), 471. 
Cage, J. (1961) Forerunners of modern music. Silence, pp.62-66.

Cage, J. (1961) History of experimental music in the United States. Silence, pp.67-75.

Garland, P. (1984). James Tenney. Soundings 13: The Music of James Tenney, 3.

Goldstein, M. (1984). Some glimpses of Jim Tenney. Soundings 13: The Music of James Tenney, 4-14.

Kasemets, U., Pearson, T., \& Monahan, G. (1984). A tradition of experimentation: James Tenney in conversation with Udo Kasemets and Musicworks. Musicworks 27, pp. 2-13, 20.

Polansky, L. (1984). The early music of James Tenney. Soundings 13: The Music of James Tenney, 114-297.

Tenney, J. (1969). Computer music experiences, 1961-1964. Electronic Music Reports, \#1. pp. 23-60.

Tenney, J. (1971). Form. Dictionary of contemporary music. John Vinton, ed. pp. 242-47.

Tenney, J. (1988). META + HODOS: A phenomenology of $20^{\text {th }}$-century musical materials and an approach to form ( $2^{\text {nd }}$ ed.). Hanover, NH: Frog Peak Music. 